\title{
New Approaches to the Assessment and Control of Electric Power Quality
}

\author{
Nafisa Solonina ${ }^{1 *}$, Konstantin Suslov $^{2}$ \\ ${ }^{1-2}$ Irkutsk National Research Technical University
}

\begin{abstract}
Currently, many loads are non-linear, and there is a trend to increase them. As is well known, nonlinear load generates harmonic components, resulting in a change of delivered power characteristics, namely, indicators degrade the supplied power quality. In turn, the deterioration of the power quality leads to its overrun. Active introduction of high-tech equipment is accompanied by an increase in the proportion of customers' special requirements for the quality of supplied electricity. In this regard, the problem of monitoring and reduction of the harmonic components is relevant. Timely and accurate measurements based on data recorders' vector quantities with high resolution offer the prospect of continuous monitoring of the quality of power supply. This paper suggests a distributed system for monitoring the power quality by the use of available PMU infrastructure to carry out continuous control of the harmonic level in different cutsets of a power system.
\end{abstract}

Keywords - Distributed monitoring of power quality, electric power systems, power quality, smart grids, voltage and current harmonics.

\section{INTRODUCTION}

Currently, due to the global shortage of energy carriers, the goal of more efficient use of the existing power sources and transmission lines is getting increasingly more important.

At the same time, however, there is a known contradiction. In order to enhance the efficiency, we use advanced industrial electronics, rectifiers, frequency transducers, inverters, etc. This increases the efficiency of the technological plants, but at the same time the supply voltage suffers from an elevated level of harmonic components in the network [1], [2].

The harmonic level in the supply voltage, according to the normative documents, is characterized by the total harmonic factor $K_{U}$, expressed as a percentage of the fundamental frequency. This, as is known, decreases the energy efficiency.

According to data [3], the share of harmonics caused by nonlinear load reaches about $20 \%$ of the total harmonics in the network.

Fig. 1 shows examples of the spectral composition of harmonic components for different types of non-linear loads (for example, aluminum smelter, railway, etc.).

Therefore, it is necessary to control harmonic distribution in various cutsets of the supply network [4], [5].

Based on the analysis of harmonic distribution we can generate signals to control the operation of frequency setting circuits: regulated capacitors, reactors, passive and active filters.

We suggest that the available phasor measurement unit PMU infrastructures used for power system monitoring can be used as the sources of primary data at different points of the network. To this end, the sensors of instantaneous values of currents and voltages which come via satellite to the control center for monitoring were installed at nodal points of the system (generators, distribution substations, consumers substations). These signals can also be used to control the power quality. It is necessary to install a smart meter for separate measurement of harmonic energy and fundamental frequency energy at the control center. This meter will receive the data on currents and voltages at different nodes of the system. Then these data are processed and a decision is made on an action through the FACTS devices on the variable (controlled) reactive components $C_{\mathrm{r}}$ and $L_{\mathrm{r}}$.
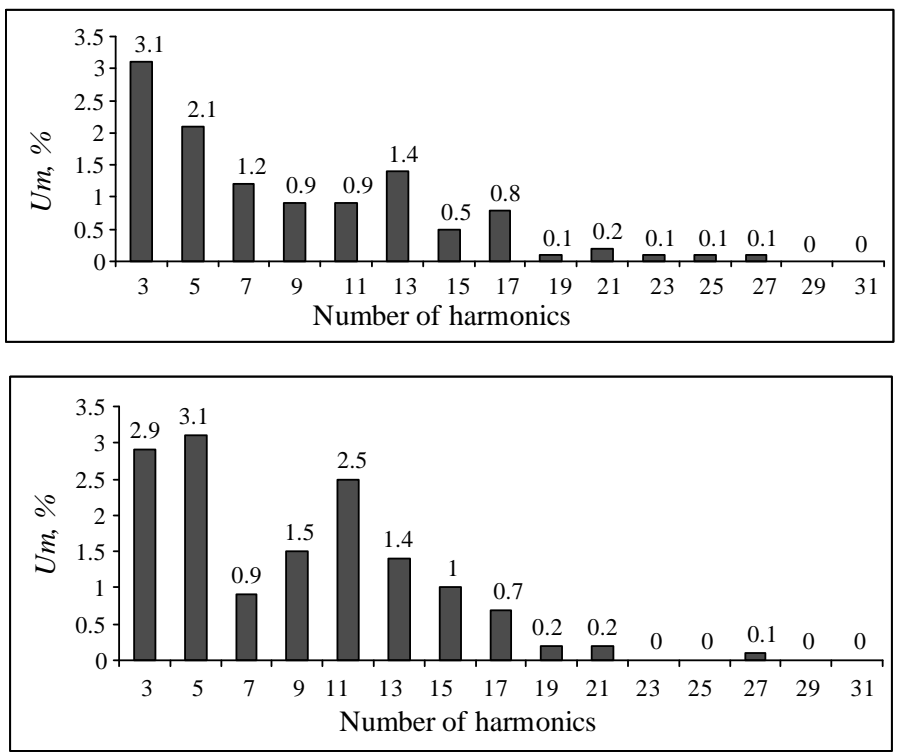

Fig. 1. Examples of the spectral composition of harmonic components for different types of non-linear loads.

${ }^{*}$ Corresponding author.

E-mail address: souslov@istu.edu 


\section{Problem StATEMENT}

Electric power system reminds of a living organism whose survivability can be provided by the devices intended for control of its parameters. These devices, on the one hand, are autonomous and, on the other hand, should be interrelated. For successful operation of an energy system, it is necessary to create a distributed system for power quality monitoring.

In this paper, the monitoring of quality is limited to the monitoring of the harmonic level in the supply voltage. To this end it is necessary to measure harmonic energy flows at different cutsets of the system in the conditions which are close to the conditions of real time.

To perform this task, there are the following two preconditions. The first is the existing PMU [6], [7] infrastructure, namely, measurement of instantaneous values of current and voltage at different cutsets and their transfer to the control center. Traditionally, these data are used for recording vector parameters of the energy system. The authors suggest using the same primary data for the determination of the energy flows of the fundamental frequency and harmonics at different cutsets using a smart meter developed by the authors [8]. The second precondition is the existence of FACTS which makes it possible to remotely change the values of reactive components of a circuit.

\section{KEY PRINCIPLES OF THE APPROACH}

Theoretically, however, it is virtually impossible to develop an algorithm for the control of parameters of several reactive components. Therefore, we suggest the use of a method of successive approximations, i.e., by randomly changing one of the parameters within some small limits, for example, a controlled capacitance, observe the system response. For an objective function we use a relationship between the power of fundamental frequency $P_{1}$ and the power of harmonics $P_{\mathrm{HH}}$, which is received from the smart meter:

$$
\frac{P_{1}}{P_{\mathrm{HH}}}=f\left(x_{1}, x_{2}, x_{3}, \ldots, x_{n}\right) \text {. }
$$

In a general case, such an objective function (1) depends on many variables where $x_{1}, x_{2}, x_{3}, \ldots, x_{n}$ are the values of reactive components that affect the frequency properties of a circuit, and $x_{1}, x_{2}$ - are variable values which can be changed remotely from the control center, and the remaining values $x_{3}, x_{4}$, etc. are quasi constant, i.e., they change at a speed of a change in the operating conditions of the system. We will consider an equivalent circuit of a typical local electricity supply system (Fig. 2). For simplicity we will assume that there is only one harmonic, for example, the third one, which is generated by both the source $e_{\mathrm{g}}$ and the nonlinear consumer $e_{3}$ , and there are two variable reactive components: capacitance $C_{\mathrm{r}}$, and inductance $L_{\mathrm{r}}$.

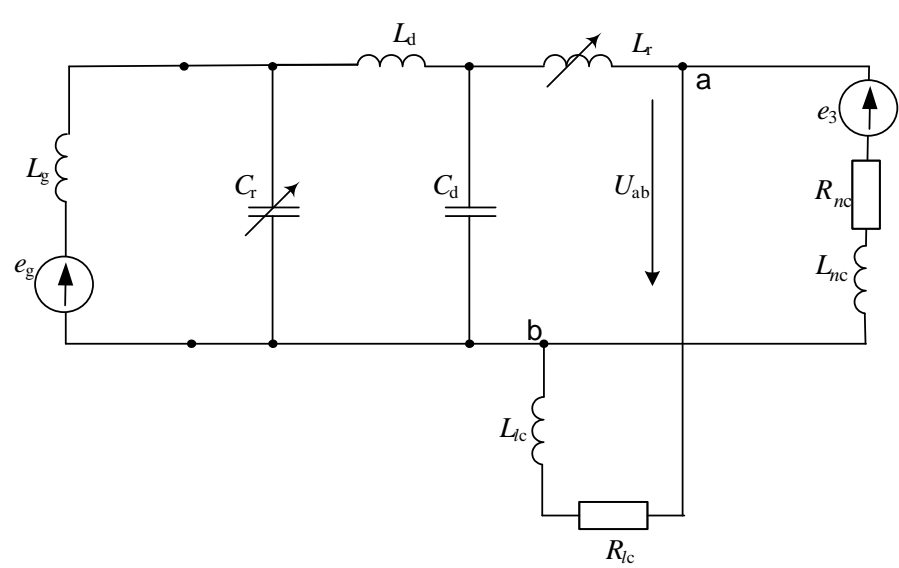

Fig. 2. An equivalent circuit of the micro-network.

To determine a principal possibility of automatically adjusting the variable values, we will consider a special case. It is necessary to ensure the highest power quality at the connection point $(\mathrm{ab})$ of a linear consumer. We will consider the inductance of generator $L_{\mathrm{g}}$, distributed capacitance $C_{\mathrm{d}}$, distributed inductance $L_{\mathrm{d}}$, and inductances of consumers $L_{n \mathrm{c}}$ and $L_{l c}$ to be constant, and the controlled capacitance $C_{\mathrm{r}}$ and controlled reactor inductance $L_{\mathrm{r}}$ to be variable.

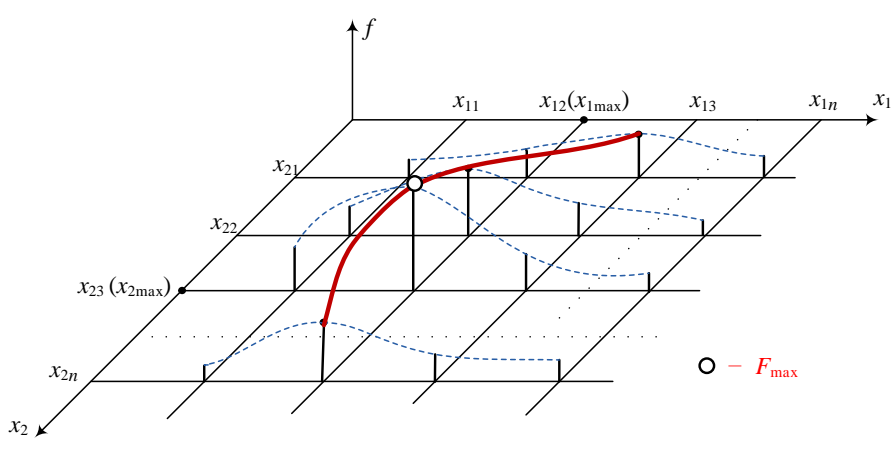

Fig. 3. Determination of the maximum objective function.

The procedure for determining the maximum objective function at two variable parameters of a circuit ( $x_{1}$ - variable capacitance; $x_{2}$-variable inductance) is presented in Fig. 3. In the presented model, we introduce the input numerical parameters of constant values $x_{3}, x_{4}$, etc., and the minimum values of a range of change in variables $x_{1}, x_{2}$. Then, we fix the first values of variable $x_{2}$ at the beginning of the established range $\left(x_{21}\right)$, and increase the variable $x_{1}$ with a constant step $\Delta x_{1}$, from the minimum value $x_{11}$ to the maximum value $x_{1 n}$ of the range where $n$ is the total number of cutsets. At each value $x_{1}$, the ratio of the fundamental frequency power to the harmonic power at points ( $a b)$ (Fig. 3) is calculated using the 
electric circuit calculation methods. We find the value $x_{\max 1}$, which corresponds to the maximum objective function (1) at given value $x_{21}$. The obtained values $x_{\max 1}, x_{21}$ and the values of the objective function $f_{\max 1}$ are entered into the memory of the logic device.

Then, we increase the variable $x_{2}$ with an increment $\Delta x_{2}$ and obtain

$$
x_{21}+\Delta x_{2}=x_{22} \text {. }
$$

Afterwards, we repeat again the operation of change in $x_{1}$ in the entire range and find $x_{1 \max 2}$ at the value $x_{22}$. Similar to the first case, we determine $f_{\max 2}$. This process is continued unless the variable $x_{2}$ reaches the upper limit of the range of its change $x_{2}=x_{2 n}$, and, correspondingly, $f_{\max n}$ is determined. The obtained data array is processed by the logic device, and the final values $x_{1 \max }, x_{2 \max }$ are determined.

Then, by comparing the calculated local maxima of the objective function $f_{\max 1}-f_{\max n}$, we determine the largest of them $-F_{\max }$, being the maximum of the objective function (1) (Fig. 3).

The values $f_{\max 1}-f_{\max n}$ are transferred to the logic device, which generates the output signal controlling the actuators. These devices in turn change the values of $x_{1}$ and $x_{2}$.

This was a preliminary determination of the optimum values of variables, as we did not consider many influencing factors, for example, not all harmonics, harmonic sources, etc. were considered.

\section{TECHNICAL IMPLEMENTATION}

When this method is technically implemented for determining the true values of variables $x_{1}$ and $x_{2}$, the above procedure should be performed by really changing the parameters. Relation (1) is fixed by the smart meter intended for separate measurements of harmonic energy and fundamental frequency energy, which makes it possible to determine the maximum values of the objective function $F_{\max }$ and the values of the local maxima $f_{\max 1}-f_{\max n}$. Accordingly, the actuators change the values of variables $x_{1}$ and $x_{2}$.

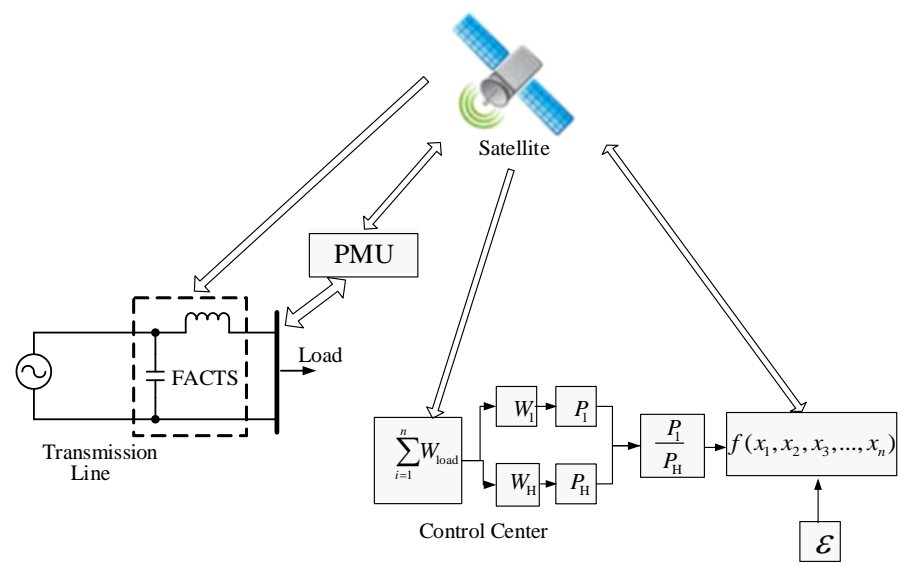

Fig. 4. Scheme of supply voltage quality control.
In order to provide the required power quality (the harmonic magnitude at the substation terminal) in the specified limits, the value of the objective function (1) should be lower than the specified value $\varepsilon$ :

$$
\frac{P_{1}}{P_{\mathrm{HH}}} \geq \varepsilon
$$

According to the European standard BS EN 50160:2010, the admissible harmonic level in the MV networks does not exceed 0.08 against the fundamental frequency, i.e., the relation should be:

$$
\frac{P_{1}}{P_{\mathrm{HH}}} \geq \frac{1}{0.08}=12.5 .
$$

Fig. 4 presents the structural scheme of the supply voltage quality control based on searching for the maximum of the objective function (1). The value of $F_{\max }$ corresponds to the minimum harmonic value in the supply voltage at the connection points. The scheme shows controlled components $x_{1}$ and $x_{2}$ : capacitance $C_{\mathrm{r}}$ and inductance $L_{\mathrm{r}}$, whose values are varied by using FACTS.

The analogue signals of instantaneous values of current $i$ and voltage $u$, at the substation terminal from the current and voltage sensors arrive at the PMU which transfers these values through the GPS satellite to the control center at the smart meter suggested by the authors [8]. The smart meter measures total energy and separately energies of fundamental frequency and harmonics multiple of the fundamental frequency. In the meter, the energy is converted to power, and at its outlet we obtain separately the signals proportional to $P_{1}$ and $P_{\mathrm{HH}}$. The signals arrive at the digital divider inlet. From the digital divider outlet, the relation $P_{1} / P_{\mathrm{HH}}$, being the objective function (1), comes to the logic device.

After the procedure of changing the values of variables $x_{1}$ and $x_{2}$, the logic device determines the maximum value of the objective function $F_{\max }$ and the values of variables $x_{1}$ and $x_{2}$, corresponding to this maximum. Herewith, the logic device generates the control signal of FACTS elements, which in turn change the values of variables $x_{1}$ and $x_{2}$ up to their optimal value.

The algorithm of searching for the objective function extremum is presented in Fig. 5. 


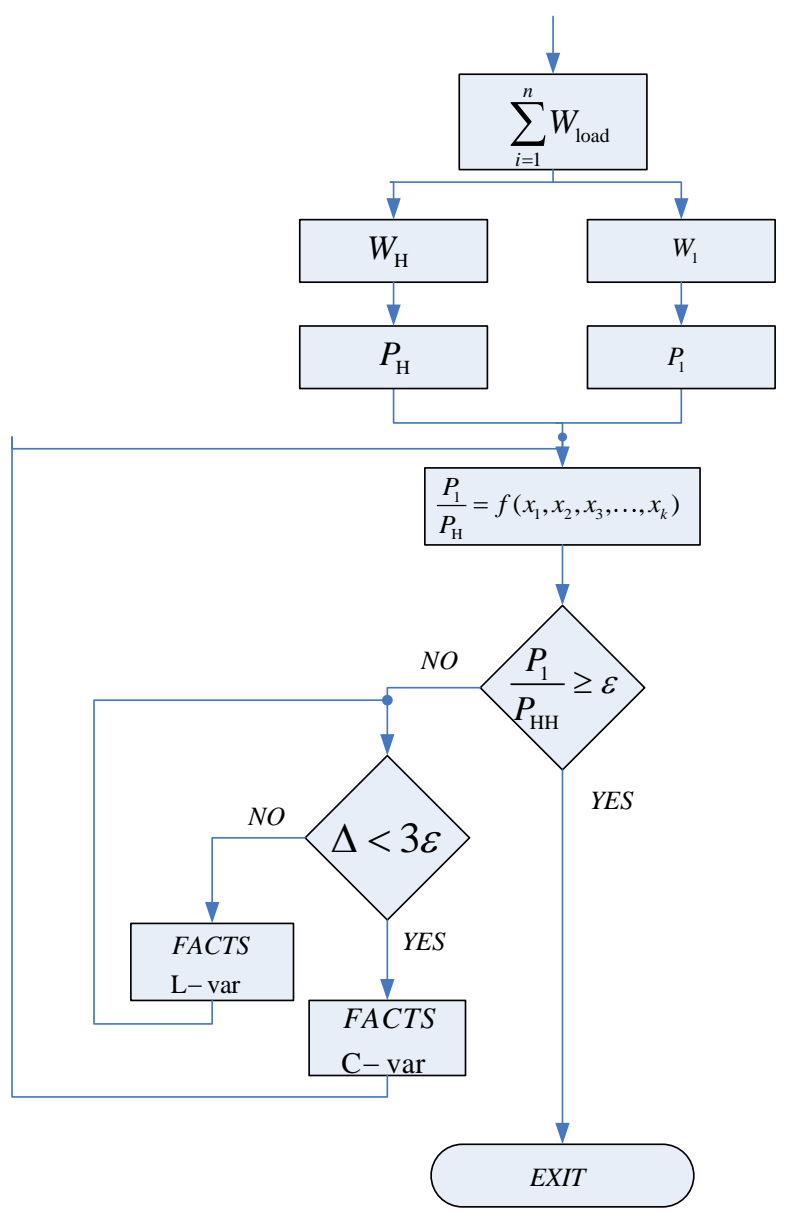

Fig. 5. Algorithm of searching for the extremum of the objective function.

The value of $P_{1} / P_{\mathrm{HH}}$ is compared with the specified value $\varepsilon$ against the condition (2). If this condition is satisfied, the power quality corresponds to the specified requirements and further correction is not needed. If the condition is not satisfied, the control signal from the logic device is supplied to FACTS through the GPS satellite to control variable parameters $x_{1}(L)$ and $x_{2}(C)$ in accordance with the earlier described procedure of successive approximations. Note that it is more reasonable to perform a rough adjustment with the help of $x_{1}(L)$, and a more accurate adjustment by using $x_{2}(C)$. The value $3 \varepsilon$ is taken as a threshold for the rough or accurate adjustment.

In particular, the reactive parameters are controlled by the static synchronous compensators (STATCOM) [9], [10]. The STATCOM is capable of controlling the output current in the whole range of capacitive and inductive current irrespective of the voltage level of the AC system. The external characteristic of the STATCOM is illustrated in Fig. 6.

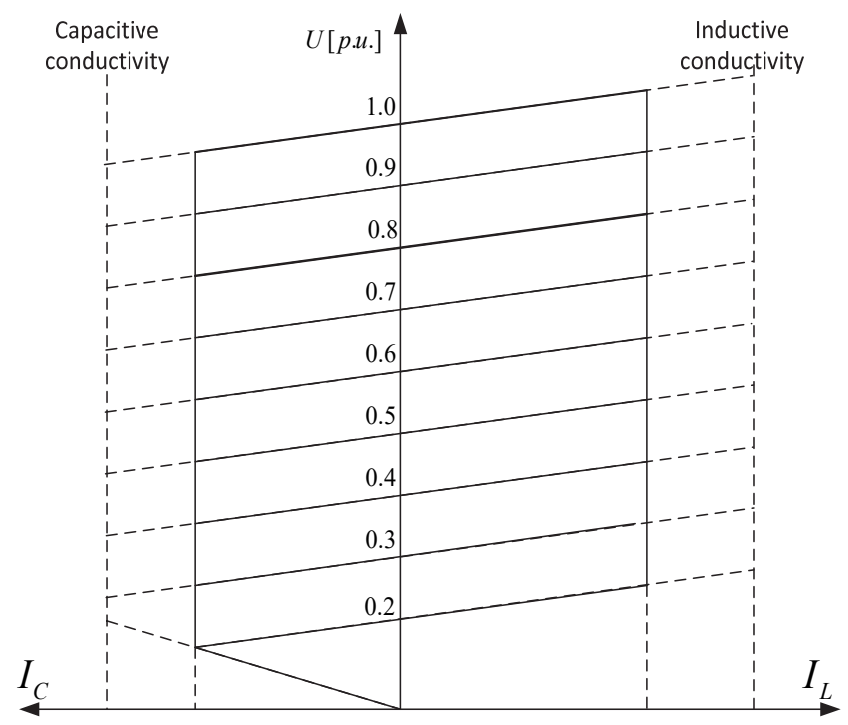

Fig. 6. External characteristic of STATCOM [9].

As compared to other devices, for example, to the static VAR compensator, the STATCOM has the following advantages [9]:

- possibility of both inductive and capacitive behaviors;

- reduction in the area occupied, since there is no need to use bulky capacitor banks and reactors for static VAR compensators;

- availability of a large dynamic control band;

- high speed and better performances during transients;

- insensitivity to harmonic resonances in the system, etc.

Since the monitoring system operates in real time, the mode of possible minimum harmonics at the connection points is continuously maintained. The described technique can be extended to more branched schemes. For this purpose it will be necessary to install a multiplexor before the meter to optimize frequency modes at many connection points.

The described procedure is performed on-line, and thus the power supply system is continuously adjusted to the mode close to the optimal one from the viewpoint of the harmonic content in the supply voltage at the selected connection points.

\section{CONCLUSION}

The methodology proposed by the authors allows to monitor the level of harmonics in real time.

The authors suggest a distributed system for monitoring the power quality by the use of available PMU infrastructure to carry out continuous control of the harmonic level in different cutsets of a power system.

A technique for the determination of the optimal values of controlled reactances is developed to reduce the harmonic level by using the FACTS devices. 


\section{REFERENCES}

[1] S. Mohibullah, and S. H. Laskar, "Power quality issues and need of intelligent PQ monitoring in the smart grid environment," in $47^{\text {th }}$ Int. Conf. in Smart Measurements for Future grids, SMFG, 2012, pp. 1-6.

http://dx.doi.org/10.1109/UPEC.2012.6398437

[2] D. Castaldo et al., "Power Quality Analysis: a Distributed Measurement System," in IEEE Power Tech Conf., 2003. http://dx.doi.org/10.1109/ptc.2003.1304437

[3] J. Arrillaga, and N. R. Watson, Power system harmonics, 2nd ed. Chichester: Wiley, 2003.

[4] K. V. Suslov, N. N. Solonina, and V. S. Stepanov, "A principle of power quality control in the intelligent distribution networks," in Int. Symp. on Smart Electric Distribution Systems and Technol., EDST, 2015, pp. 260264. http://dx.doi.org/10.1109/sedst.2015.7315218

[5] Z. H Yuan, and Y. Du, "A shunt active harmonic filter based on a voltage detection method for harmonic voltage control Electric Utility Deregulation, Restructuring and Power Technologies," in IEEE Int. Conf. on Electric Utility Deregulation, Restructuring and Power Technol., vol. 2, 2004, pp. 763-768. http://dx.doi.org/10.1109/DRPT.2004.1338085

[6] Z. Wu, L. T. Zora and A. G. Phadke, "Simultaneous transmission line parameter and PMU measurement calibration," in IEEE Power \& Energy Society General Meeting, 2015. http://dx.doi.org/10.1109/PESGM.2015.7286115

[7] S. Nuthalapati and S. Phadke, "A Managing the Grid: Using Synchrophasor Technology," Power and Energy Magazine, IEEE, vol. 13, Issue 5, 2015. http://dx.doi.org/10.1109/MPE.2015.2435596

[8] A. S. Smirnov, N. N. Solonina, and K. V. Suslov, "Separate measurement of fundamental and high harmonic energy at consumer inlet - a way to enhancement of electricity use efficiency," in Proc. Int. Conf. on Power System Technol., POWERCON, 2010. http://dx.doi.org/10.1109/powercon.2010.5666617

[9] V. K. Sood, HVDC and FACTS Controllers: Applications of Static Converters in Power Systems, Springer, 2004

[10] W. Sung-Min et al., "The distribution STATCOM for reducing the effect of voltage sag and swell," in 27th Annu. Conf. of the IEEE Industrial Electronics Society, 2001, vol. 2, pp. 1132-1137.

http://dx.doi.org/10.1109/iecon.2001.975939 